\title{
Safe Patients, Smart Hospitals: How One Doctor's Checklist Can Help Us Change Health Care from the Inside Out
}

\author{
P. Pronovost and E. Vohr \\ New York, NY: Hudson Street Press, 2010, 282 pages, \$25.95
}

In the United States it is estimated that because of a lack of knowledge or expertise, thousands of physicians do not provide the currently recommended treatments to millions of patients seeking medical care. Even more alarming, medical errors are the principal cause of death in hundreds of thousands of patients each year. Well-trained and wellintentioned physicians leave instruments in patients, overdose with medication, operate on the wrong side of the body, or even treat medical conditions that patients do not actually have. One cause of medical error is the lack of standardization in performing everyday procedures using evidence-based training with the latest medical research. Too often, medicine relies on training based on the age-old adage "see one, do one, teach one," in which medical students and residents obtain their knowledge from one or two physicians without knowing whether that learning is correct. As medical training goes on, these health care providers pass on incorrect procedures unchecked. More disturbingly, an outdated hospital culture can perpetuate medical errors when physicians-in-training are discouraged from asking questions for fear of being humiliated, and true teamwork between attending physicians, residents, and nurses does not exist.

Safe Patients, Smart Hospitals chronicles Dr. Peter Pronovost's journey to improve patient safety and prevent unnecessary patient deaths. Starting at Johns Hopkins Hospital with the simple improvement of central line placement by putting together a checklist to follow, Dr. Pronovost's work led to the development of patient care improvements that have been adopted in hospitals across the country and internationally.

At Johns Hopkins Hospital, central line catheters were not a standardized procedure, each being placed differently depending on the physician. Although there existed comprehensive Centers for Disease Control and Prevention guidelines explaining how to properly place central line catheters and prevent infections, it ran 120 pages long. Furthermore, the instructions were convoluted and unclear. As a result, only $30 \%$ of patients with central line catheters were treated in accordance with these guidelines. With such poor compliance, infections remained so high that clini-

COPYRIGHT $\odot 2011$ by the Society of Nuclear Medicine, Inc. DOI: 10.2967/jnumed.110.082537 cians accepted nosocomial infections as inevitable. Dr. Pronovost believed that the hospital-acquired infections at Johns Hopkins were preventable and, thus, created an easyto-follow 5-step checklist for placing a central catheter line. After the so-called "Pronovost's Checklist" had been instituted, central line infection rates dropped dramatically to zero in $1 \mathrm{y}$, with an estimated health care saving of \$2 million. Pronovost's Checklist is one of the many checklists the author created to make the hospital a safer place for patients. Two other highly successful checklists were created to decrease ventilator-associated pneumonia and surgical site infections. To ensure the widespread use of these checklists, Dr. Pronovost implemented creative yet radical ways to incorporate nurses, staff, and even the patient's family into monitoring the doctors for compliance.

The idea of creating a simple checklist came from the aviation industry. Dr. Pronovost noticed the striking similarity between aviation and medicine. A pilot and a physician are both in charge of making critical decisions and, ultimately, are responsible for their passengers or patients' safety. Both professions require perfection, or else death can easily occur as a result of errors. However, clear differences distinguish the two fields. In the aviation industry, humans are generally seen as fallible. As a result, the aviation industry created checklists to minimize human error. On the other hand, the medical field has had a hard time accepting the idea that physicians make mistakes. In addition, keeping track of errors is more complicated in the medical field than in the aviation industry. Generally speaking, passengers flying on an airplane are healthy whereas patients in a hospital are sick. Therefore, determining whether a patient died from medical error or natural causes is often difficult. Moreover, medical errors are often not even reported for fear of lawsuits. Furthermore, the amount of medical information required of a physician cannot be compared with that of a pilot in order to safely operate a plane. Also as an impediment, doctors are often erroneously taught that guidelines are for simple physicians, not expert clinicians. Despite such differences, Dr. Pronovost appreciated the similarity between the two fields and used the idea of a simple checklist.

Another underlying problem that was tackled by Dr. Pronovost was the toxic culture that existed in the medical 
field. The author's safety team reviewed the cause of medical errors and found that in nearly $90 \%$ of medical errors, one of the team members knew something was wrong but never spoke up or, even worse, was ignored. There was a clear breakdown in communication and poor teamwork that was not being acknowledged. One extreme example was found in the operating rooms at Johns Hopkins Hospital. The operating room exhibits one of the most stringent hierarchical systems in a hospital headed by the chief surgeon. With absolute authority residing in the chief surgeon, concerns raised by any other member of the surgical team, including other physicians, were often ignored. All decisions were ultimately left to the discretion of the surgeon in charge. Therefore, medical errors persisted and patient care suffered. To reduce medical errors, Dr. Pronovost recognized that a cultural change was needed to overcome the resistant attitudes and to improve teamwork and communication. Dr. Pronovost's team placed greater emphasis on educating medical providers on how teams make better and wiser decisions than a single person in reducing medical errors. Dr. Pronovost's program helped medical providers realize why their opinions were important and helpful. Additionally, the program asked everyone, including nurses, physicians, social workers, pharmacists, and administrators, to actively participate to ensure patient safety. This change promoted ownership and, in doing so, spread the responsibility of patient safety to everyone in the hospital and not just to physicians. With each safety issue that was addressed, checklists and solutions to problems were created as a team. In addition, questionnaires and statistics became important to document improvement and to show that active participation made a difference.

Safe Patients, Smart Hospitals is an inspiring and thoughtprovoking book on improving patient safety that began with a simple checklist at Johns Hopkins Hospital. Throughout his journey, Dr. Pronovost encountered numerous resistances and opponents to his work. The author worked hard and was able to stay focused on improving the quality and safety of care delivered to patients. As a result of his work, Time magazine named Dr. Pronovost one of the 100 World's Most Influential People in 2008. Today, hospitals in all 50 states are using Dr. Pronovost's safety program. Guided with a simple vision and plan, Dr. Pronovost's dream of making hospitals a safer place for patients is becoming true.

\author{
Hye Ryong Kim \\ Robert Matthews* \\ "Stony Brook University Hospital \\ HSC Building, Level 4, Room 120 \\ Stony Brook, NY 11794 \\ E-mail: robert.matthews@stonybrook.edu
}

